\title{
Fermion or Boson Dark Matter?
}

\author{
Bruce Hoeneisen \\ Universidad San Francisco de Quito, Quito, Ecuador \\ Email: bhoeneisen@usfq.edu.ec
}

How to cite this paper: Hoeneisen, B. (2020) Fermion or Boson Dark Matter? International Journal of Astronomy and Astrophysics, 10, 203-223.

https://doi.org/10.4236/ijaa.2020.103011

Received: July 30, 2020

Accepted: August 31, 2020

Published: September 3, 2020

Copyright $\odot 2020$ by author(s) and Scientific Research Publishing Inc. This work is licensed under the Creative Commons Attribution International License (CC BY 4.0).

http://creativecommons.org/licenses/by/4.0/

\begin{abstract}
We measure properties of dark matter in four well motivated scenarios: fermions with ultra-relativistic thermal equilibrium (URTE), bosons with URTE, fermions with non-relativistic thermal equilibrium (NRTE), and bosons with NRTE. We attempt to discriminate between these four scenarios with studies of spiral galaxy rotation curves, and galaxy stellar mass distributions. The measurements show evidence for boson dark matter with a significance of $3.5 \sigma$, and obtain no significant discrimination between URTE and NRTE.
\end{abstract}

\section{Keywords}

Dark Matter, Free-Streaming, Galaxy Mass Distribution, Spiral Galaxy

\section{Introduction}

Non-relativistic dark matter in the early universe has a density $\rho_{h}(a)$ that scales as $a^{-3}$, and a particle root-mean-square (rms) velocity $v_{h r m s}(a)$ that scales as $a^{-1}$, where $a$ is the expansion parameter. (Throughout, the sub-index " $h$ " stands for the halo of dark matter.) Note that $v_{h r m s}(a) / \rho_{h}(a)^{1 / 3}$ is an adiabatic invariant independent of $a$. Now consider a free observer in a density peak. This observer feels no gravity, observes dark matter expanding adiabatically, reaching maximum expansion, and then collapsing adiabatically into the core of a galaxy. Note that adiabatic expansion implies

$$
\frac{v_{h r \mathrm{~ms}}(a)}{\rho_{h}(a)^{1 / 3}}=\frac{v_{h \mathrm{rms}}(1)}{\left(\Omega_{c} \rho_{\text {crit }}\right)^{1 / 3}}=\frac{\sqrt{3}\left\langle v_{r h}^{2}\right\rangle^{1 / 2}}{\rho_{h}(r \rightarrow 0)^{1 / 3}},
$$

where $\sqrt{3}\left\langle v_{r h}^{2}\right\rangle^{1 / 2}$ is the root-mean-square velocity of dark matter particles in the core of the galaxy, and $\rho_{h}(r \rightarrow 0)$ is the density of dark matter in the core of the galaxy. (We use the standard notation in cosmology as defined in [1].) The interest in Equation (1) lies in the ability to measure $\left\langle v_{r h}^{2}\right\rangle^{1 / 2}, \rho_{h}(r \rightarrow 0)$, 
and $v_{h r m s}(1)$ by fitting spiral galaxy rotation curves.

The adiabatic invariant $v_{h r m s}(1)$ remains constant so long as the mean number of dark matter particles per orbital remains constant, as expected for non-interacting dark matter. The issue of possible phase-space dilution due to galaxy structure formation appears to be secondary, since measurements of $v_{h \mathrm{rms}}(1)$ in 10 galaxies of the THINGS sample [2], and 46 different galaxies in the SPARC sample [3], obtain results consistent within statistical and systematic uncertainties [4] [5]. We therefore interpret $v_{h r m s}(1)$ to be of cosmological origin: it determines the ratio of dark matter temperature $T(a)$ to mass $m_{h}$ in the early universe. Note that dark matter becomes non-relativistic at expansion parameter $a \approx a_{h \mathrm{NR}}^{\prime} \equiv v_{h \mathrm{rms}}(1) / c$.

To obtain $T(a)$ and $m_{h}$ separately, we need one more constraint, e.g. the chemical potential $\mu$ of dark matter. It turns out that the measured value of $v_{h r m s}(1)$ corresponds to thermal equilibrium between dark matter and the standard model sector in the early universe if $\mu=0$. This result is either a coincidence, or strong evidence that the chemical potential of dark matter has the very special value $\mu=0$.

Thus we arrive at the following scenario: in the early universe dark matter is in diffusive and thermal equilibrium with the standard model sector, and decouples (from the standard model sector, and from self annihilation) while still ultra-relativistic. In particular, we assume that dark matter has zero chemical potential $\mu$. This no freeze-in and no freeze-out scenario is the result of measurements presented in [4] [5] [6] [7] [8]. A convenient overview of these studies, and a discussion of the (apparent?) disagreements with current limits, are presented in [9].

In the no freeze-in and no freeze-out scenario, the ultra-relativistic dark matter is in ultra-relativistic thermal equilibrium (URTE), either Fermi-Dirac, or Bose-Einstein. As the universe expands and cools, dark matter becomes non-relativistic. The momentum distribution of the non-relativistic dark matter particles approaches non-relativistic thermal equilibrium (NRTE) due to dark matter-dark matter elastic interactions [10]. If these interactions are sufficiently strong, dark matter acquires the NRTE distribution, either Fermi-Dirac or Bose-Einstein. If, however, the dominant dark matter-dark matter interaction is gravity, then the time constant to approach NRTE is much greater than the age of the universe, and non-relativistic dark matter retains the URTE distribution. Summaries of NRTE and URTE are presented in Appendix A and Appendix B, respectively.

The purpose of the present study is to try to discriminate between these four alternatives for non-relativistic dark matter with zero chemical potential: fermions with URTE, bosons with URTE, fermions with NRTE, or bosons with NRTE. We investigate the following observables: spiral galaxy rotation curves, and galaxy stellar mass distributions at large redshift $z$, and compare the results with the expectations of the no freeze-in and no freeze-out assumption. 
In the following sections we study the dark matter equation of state, spiral galaxy rotation curves, dark matter free-streaming, the no freeze-in and no freeze-out scenario, and galaxy stellar mass distributions, and, finally, present the conclusions.

\section{Dark Matter Equation of State}

We analyze rotation curves of galaxies in the Spitzer Photometry and Accurate Rotation Curves (SPARC) catalog [3]. An example is presented in Figure 1. Let $v_{\text {tot }}(r) \equiv v(r)$ be the velocity of a test particle in a circular orbit of radius $r$ in the plane of the galaxy. $v(r)$ has contributions $v_{b}(r)$ from baryons, and $v_{h}(r)$ from dark matter:

$$
\begin{gathered}
v(r)^{2}=v_{b}(r)^{2}+v_{h}(r)^{2}, \\
v_{b}=\sqrt{\left|v_{\text {gas }}\right| v_{\text {gas }}+\Upsilon_{\text {disk }}\left|V_{\text {disk }}\right| V_{\text {disk }}+\Upsilon_{\text {bulge }}\left|V_{\text {bulge }}\right| V_{\text {bulge }}} .
\end{gathered}
$$

$V_{\text {disk }}$ and $V_{\text {bulge }}$ are stellar contributions to the rotation velocity inferred from the $3.6 \mu \mathrm{m}$ SPARC photometry [3], assuming a stellar mass-to-light ratio $1 M_{\odot} / L_{\odot}$. The mass-to-light ratios of stars in the disk and bulge, in units of $M_{\odot} / L_{\odot}$, are taken to be $\Upsilon_{\text {disk }} \equiv \Upsilon_{*}$ and $\Upsilon_{\text {bulge }}=1.4 \Upsilon_{*}$ respectively [3]. Estimates of $\Upsilon_{*}$ range from 0.5 to 0.2 , see the discussion in Reference [3]. We take the stellar mass-to-light ratio equal to its fitted average $\Upsilon_{*}=0.32$ [5], except for galaxies F574-1 and UGC11914 for which we take $\Upsilon_{*}=0.2$ as in [5].

Two differential equations of interest to dark matter are Newton's equation for gravity, and the equation of conservation of the radial component of the momentum of the dark matter particles [4]:

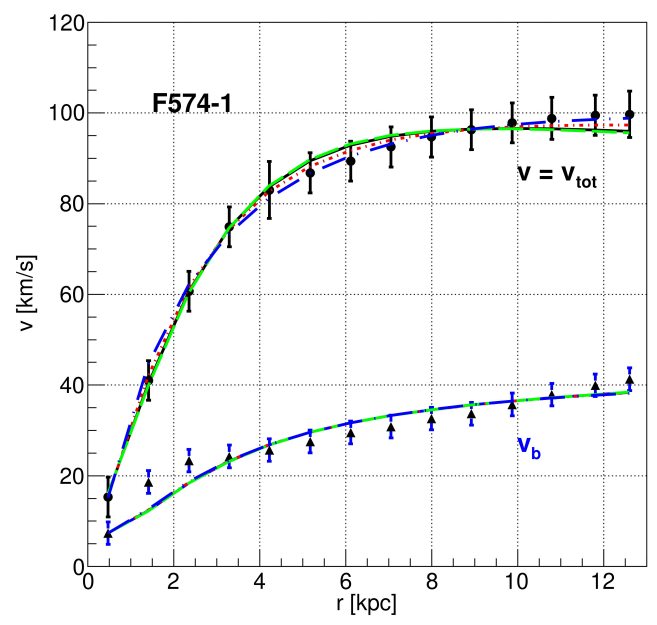

Figure 1. Rotation velocities $v(r) \equiv v_{\text {tot }}(r)$ of galaxy F574-1 as a function of the distance $r$ from the center of the galaxy, taken from the SPARC catalog [3]. $v_{b}$ is the contribution from baryons. The curves are fitted numerical integrations for fermions with URTE (black continuous lines), bosons with URTE (red dot-short dash), fermions with NRTE (green long dash), and bosons with NRTE (blue dot-long dash). The numerical integrations start with $\mu^{\prime}=0$ at $r \rightarrow 0$. The $\chi^{2}$ 's are presented in Table 2. 


$$
\begin{gathered}
\frac{1}{r^{2}} \frac{\mathrm{d}\left(r^{2} g_{h}\right)}{\mathrm{d} r}=4 \pi G \rho_{h}, \\
\frac{\mathrm{d} P_{h}}{\mathrm{~d} r}=-\rho_{h} g\left(1-\kappa_{h}\right) .
\end{gathered}
$$

$g=v^{2} / r=g_{h}+g_{b}$ is the gravitation field, and $\kappa_{h}$ is a correction due to dark matter rotation. The definition of pressure $P_{h}$, for collisional or collision-less dark matter, is presented at the end of Appendix B. We take $\kappa_{h}=0.15_{-0.15}^{+0.35} \quad$ [4].

From $g_{h i}$ at every measured radii $r_{i}$, and the difference equation corresponding to (4), we obtain $\rho_{h i}$ at some point in each interval $r_{i}<r<r_{i+1}$. From the difference equation corresponding to (5), starting at $r_{\text {max }}$, we obtain the accumulated pressure $P_{h i}$ down to radii $r_{i}$. The root-mean-square of the radial component of the velocities of dark matter particles, $v_{r h r m s}\left(r_{i}\right)=\left[2 P_{h i} /\left(\rho_{h i}+\rho_{h(i-1)}\right)\right]^{1 / 2}$, is plotted in Figure 2 for galaxy F574-1.

In the case of thermal equilibrium, either URTE or NRTE, the equation of state of dark matter has the form $P_{h}(r) / \rho_{h}(r)=f\left(T_{h}, \mu(r)\right)$, see Appendix A and Appendix B. $T_{h}$ is the dark matter temperature, and $\mu(r)$ is the dark matter chemical potential. $f\left(T_{h}, \mu(r)\right)=v_{r h \mathrm{rms}}^{2}(r)=v_{h \mathrm{rms}}^{2}(r) / 3$, where $v_{h \mathrm{rms}}^{2}(r)$ is the mean velocity squared of the dark matter particles (see Appendix B). Excellent fits to galaxy rotation curves are obtained assuming thermal equilibrium [4] [5]. In thermal equilibrium, $T_{h}$ is a constant independent of $r$, while $\mu(r)$ becomes more negative with increasing $r$ due to the gravitational field.

We define $\mu^{\prime} \equiv \mu /\left(k T_{h}\right)$. For $\mu^{\prime} \ll 0, f\left(T_{h}, \mu(r)\right)$ becomes independent of $r$. For $\mu^{\prime}(0)=0$ in the core of the galaxy, $f\left(T_{h}, \mu(r)\right)$ increases (decreases) at the first two or three measured $r_{i}$ for fermions (bosons), see Figure 2. To distinguish fermion from boson dark matter, we hope to measure this increase or decrease of $v_{r h r m s}\left(r_{i}\right)$. However, as seen in Figure 2 and Figure 3, $v_{r h r m s}$ is consistent with being a constant, with large uncertainties that prevent us from distinguishing fermions from bosons by this direct method.

\section{Fits to Spiral Galaxy Rotation Curves}

To gain sensitivity, we integrate numerically (4) and (5), and two similar equations for baryons [4], starting at $r_{\min }$. To start these integrations we need four boundary conditions. We also require the equation of state of dark matter to obtain $\rho_{h}$ given $P_{h}$ (see Appendix A and Appendix B). In References [4] [5] we use these boundary conditions: $\rho_{h}\left(r_{\min }\right), v_{r h r m s}\left(r_{\min }\right), \rho_{b}\left(r_{\min }\right)$, and $v_{r b \mathrm{rms}}\left(r_{\min }\right)$. We vary these four parameters to minimize the $\chi^{2}$ between the measured and calculated rotation curves. The mass $m_{h}$ of the dark matter particles is kept fixed in these fits.

In the present analysis we use the following equivalent set of four boundary conditions: $a_{h \mathrm{NR}}^{\prime}\left(r_{\min }\right), \mathbb{V} \equiv \sqrt{3 k T_{h} /\left(m_{h} c^{2}\right)}, \quad \rho_{b}\left(r_{\min }\right)$, and $v_{r b \mathrm{rms}}\left(r_{\min }\right)$. We are therefore able to keep $\mu^{\prime}\left(r_{\min }\right)=0$ fixed in the fits (for bosons we need to avoid the singularity at $r_{\min }$, so we start the integration with $\mu^{\prime}\left(r_{\min }\right)=-0.1$, 
$-0.01,-0.001$, or -0.0001$)$. Furthermore, fitting $a_{h \mathrm{NR}}^{\prime}\left(r_{\min }\right)$, and calculating $a_{h \mathrm{NR}}^{\prime}(r)$, we are able to extrapolate to $r \rightarrow 0$ and obtain $a_{h \mathrm{NR}}^{\prime}$ in the core of the galaxy. In the present analysis, we free the first measured rotation velocity $v\left(r_{\text {min }}\right)$. The parameters $a_{h \mathrm{NR}}^{\prime}\left(r_{\text {min }}\right), \mathbb{V}, \rho_{b}\left(r_{\text {min }}\right), v_{r b r m s}\left(r_{\text {min }}\right)$, and $v\left(r_{\text {min }}\right)$ are varied to minimize the $\chi^{2}$.

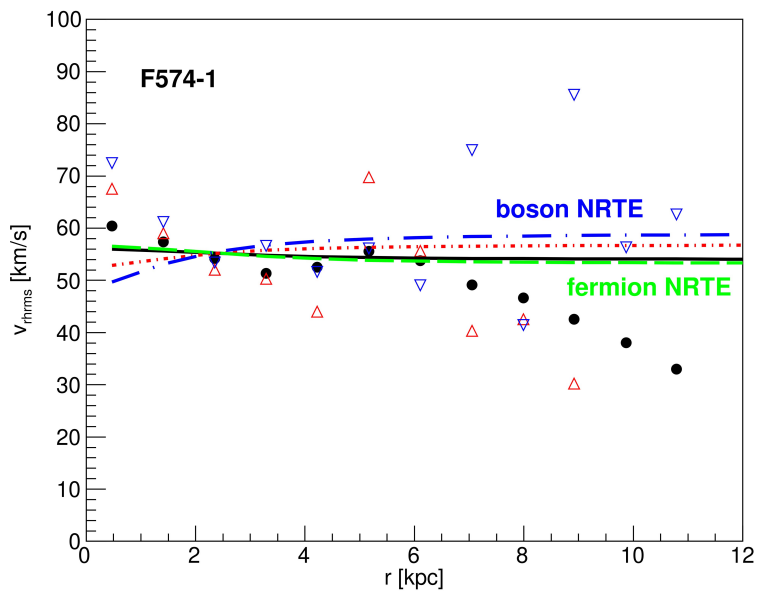

Figure 2. Equation of state of dark matter, $\left[P_{h}(r) / \rho_{h}(r)\right]^{1 / 2}=v_{r h m s}(r)$, obtained directly from the measured rotation velocities of galaxy F574-1 presented in Figure 1 (black dots). Displacing the measured velocities $v\left(r_{i}\right)$ with random Gaussian distributions with their experimental uncertainties obtains the triangles. The theoretical equations of states, obtained from the fits of numerical integrations, are indicated with lines. The line colors and styles are as in Figure 1.
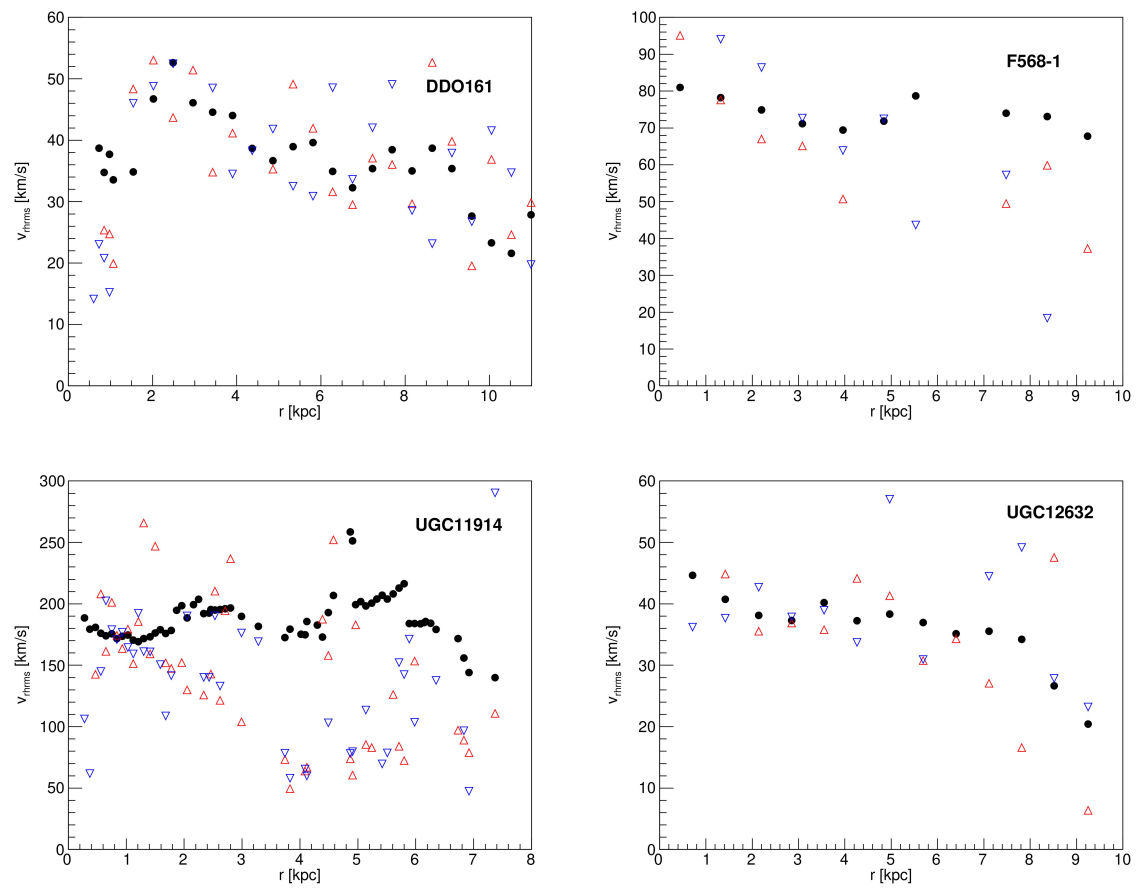

Figure 3. Equation of state of dark matter, $\left[P_{h}(r) / \rho_{h}(r)\right]^{1 / 2}=v_{r h m s}(r)$, of several galaxies in the SPARC sample [3]. Marker symbols are as in Figure 2. 
Fits for galaxy F574-1 are presented in Table 1, and Figure 1, Figure 2, and Figure 4. The $\chi^{2}$ of the fits, as a function of $\mu^{\prime}$, are presented in Figure 5. Note, in Figure 5, that the fits for $\mu^{\prime} \equiv \mu^{\prime}(r \rightarrow 0)=0$ favor bosons over fermions, but the difference in $\chi^{2}$ is not statistically significant. Note also that the $\chi^{2}$ increases for fermions as $\mu^{\prime}\left(r_{\min }\right)$ is raised above zero, so we obtain the following lower bound to the mass of dark matter particles if fermions: $48 \mathrm{eV}$ at $3 \sigma$ (or 99.7\%) confidence, similarly to what we obtained in [4]. For bosons the lower bounds are the actual measurements summarized in Table 4. Finally, note in Figure 5 that the four dark matter scenarios studied in this article are extreme and well motivated cases of interest.

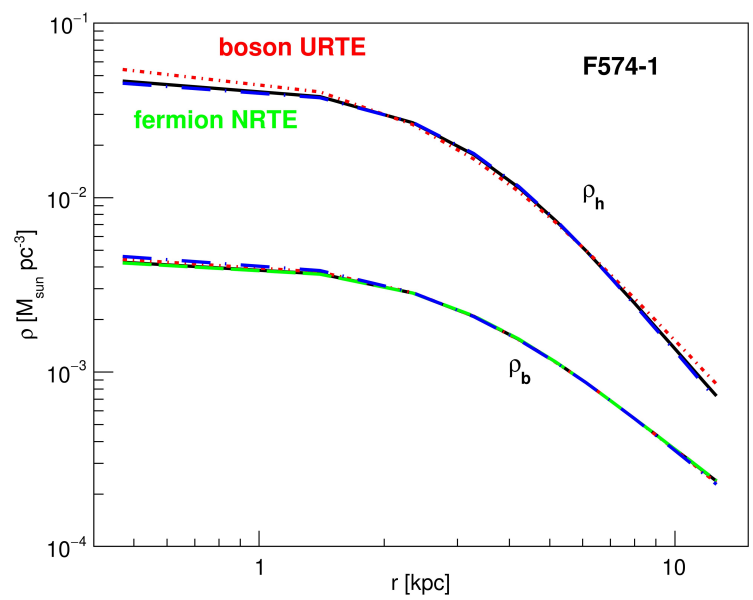

Figure 4. Densities of dark matter $\rho_{h}(r)$ and baryons $\rho_{b}(r)$ of galaxy F574-1 obtained from the numerical integrations presented in Figure 1 . The line colors and styles are as in Figure 1.

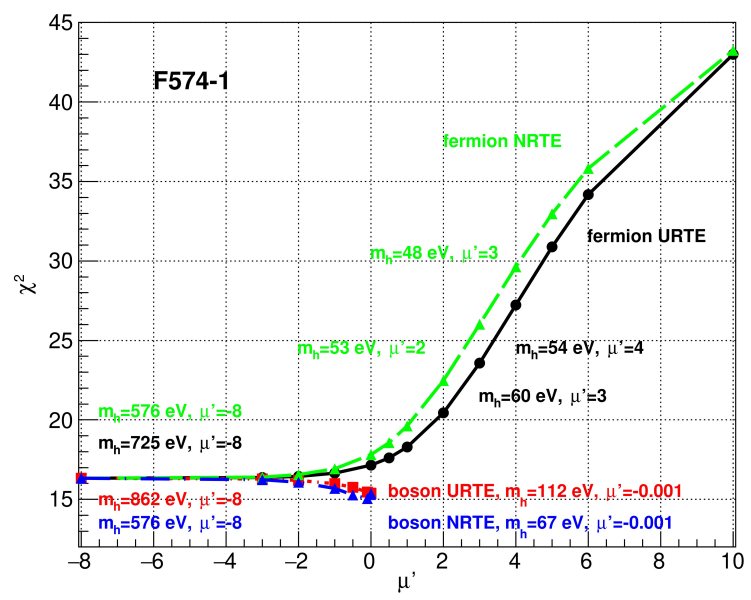

Figure 5. $\chi^{2}$ of fits to rotation curves of galaxy F574-1 for several $\mu^{\prime} \equiv \mu /\left(k T_{h}\right) . \quad \mu$ is the dark matter chemical potential in the core of the galaxy. Boson dark matter is preferred by the fits, but the difference of $\chi^{2}$ between NRTE fermions and NRTE bosons at $\mu^{\prime}=0$, i.e. $\Delta \chi^{2}=17.8-15.4=2.4$, is not statistically significant. From these fits we find that the mass of fermion dark matter particles is greater than $48 \mathrm{eV}$ with $99.7 \%$ confidence. For all fits in the figure, $a_{h \mathrm{NR}}^{\prime}$ lies in the range $1.8 \times 10^{-6}$ to $3.4 \times 10^{-6}$. 
Table 1. Fits to rotation curves of galaxy F574-1 in four dark matter scenarios. $\mu^{\prime}\left(r_{\min }\right)=0$ for fermion dark matter, $\mu^{\prime}\left(r_{\min }\right)=-0.001$ for boson dark matter, $\kappa_{b}=0.98, \kappa_{h}=0.15$, $\Upsilon_{*}=0.2$, and $\Delta v_{b}\left(r_{i}\right)=2.5 \mathrm{~km} / \mathrm{s} . \mathbb{V} \equiv \sqrt{3 k T_{h} /\left(m_{h} c^{2}\right)}$. The $\chi^{2}$ of the fits are presented in Table 2 and Figure 5. $a_{h \mathrm{NR}}^{\prime}$ has been extrapolated to $r \rightarrow 0$. Uncertainties are statistical. The systematic uncertainties of $a_{h \mathrm{NR}}^{\prime}$ are presented in Table 3.

\begin{tabular}{cccccc}
\hline Scenario & $10^{6} a_{h \mathrm{NR}}^{\prime}$ & $10^{4} \mathrm{~V}$ & $\left\langle v_{r b}^{2}\left(r_{\text {min }}\right)\right\rangle^{1 / 2}$ & $10^{3} \rho_{b}\left(r_{\text {min }}\right)$ & $v\left(r_{\text {min }}\right)$ \\
\hline Fermion URTE & $2.77 \pm 0.15$ & $164 \pm 2$ & $11.4 \pm 0.9$ & $2.3 \pm 0.4$ & $17.4 \pm 4.0$ \\
Boson URTE & $2.40 \pm 0.15$ & $169 \pm 2$ & $11.3 \pm 0.8$ & $2.4 \pm 0.4$ & $17.0 \pm 4.0$ \\
Fermion NRTE & $2.84 \pm 0.15$ & $3.07 \pm 0.06$ & $11.5 \pm 0.9$ & $2.3 \pm 0.4$ & $17.6 \pm 4.1$ \\
Boson NRTE & $1.56 \pm 0.12$ & $3.45 \pm 0.08$ & $11.1 \pm 0.8$ & $2.5 \pm 0.5$ & $16.7 \pm 3.9$ \\
\hline
\end{tabular}

A summary of fits to the rotation curves of several galaxies, selected for their very well measured $v_{\text {flat }}$ and core, and reaching deep into the core, are presented in Table 2. The quality of these fits justifies the assumption of thermal equilibrium. For fermions with URTE we plot the distribution of the measured $a_{h \mathrm{NR}}^{\prime}$ in Figure 6. These $a_{h \mathrm{NR}}^{\prime}$ are consistent with each other, within statistical and systematic uncertainties, as shown in Table 3. The mass $m_{h}$ of dark matter particles is a function of $a_{h \mathrm{NR}}^{\prime}$ and $\mu^{\prime}$, see Appendix A and Appendix B. Therefore, if $a_{h \mathrm{NR}}^{\prime}$ is equal in the core of relaxed steady state galaxies, we might expect that $\mu^{\prime}$ is also equal in these galaxies, and hence is also of cosmological origin. If we set $\mu^{\prime}=0$, each galaxy allows an independent measurement of $m_{h}$. The distribution of $m_{h}$ for fermions with URTE is shown in Figure 7. The consistency of these measurements is evidence that $\mu^{\prime}$ is indeed equal (within uncertainties) for all studied galaxies.

Let us consider the systematic uncertainties in Table 3. Galaxies DDO161 and UGC11914 have $\rho_{h}(0) \approx \rho_{b}(0)$ in the core, so the systematic uncertainties due to the uncertainty of the mass/luminosity ratio $\Upsilon_{*}$, is large. Galaxies F568-1, F574-1, UGC0024, and UGC12632 have $\rho_{h}(0)>4 \rho_{b}(0)$ [5], so the dominant systematic uncertainty is due to the unknown dark matter rotation parameter $k_{h}$. In addition to the known systematic uncertainties listed in Table 3, there are unknown systematic uncertainties including non-steady state galaxies, extraneous features of the rotation curves, phase space dilution, and systematic uncertainties of the observations. A summary of results for all galaxies listed in Table 2 is presented in rows "Spiral galaxies" of Table 4. In view of our incomplete understanding of systematic uncertainties, we assign the standard deviation of the distributions in Table 2 as the total uncertainties in rows "Spiral galaxies" in Table 4.

Let us examine the $\chi^{2}$ 's in Table 2. There is generally a preference for boson dark matter over fermion dark matter, but the difference $\Delta \chi^{2}$ is not statistical- 
ly significant for individual galaxies, except for UGC11914, see Figure 8. However, the core of UGC11914 is not dominated by dark matter, so the results from this galaxy need to be taken with caution. In Table 2 we have marked the galaxies with dark matter dominating the core, i.e. $\rho_{h}\left(r_{\min }\right)>4 \rho_{b}\left(r_{\min }\right)$ [5], and for these galaxies we have presented the sums of $\chi^{2}$ in the last row. We note that $\sum \Delta \chi^{2}=8.8$ for URTE, and $\sum \Delta \chi^{2}=11.5$ for NRTE. Thus, we have a $3.0 \sigma$ or $3.4 \sigma$ preference for bosons over fermions. We note, in Table 2 , that the standard deviation of $a_{h \mathrm{NR}}^{\prime}$ is smaller for galaxies with dark matter dominating the core.

Table 2. Summary of fits to galaxy rotation curves in four scenarios with $\mu^{\prime}=0 . a_{h \mathrm{NR}}^{\prime}$ has been extrapolated to $r \rightarrow 0$. The data is from the SPARC sample [3]. Uncertainties are statistical. ${ }^{*}$ indicates 9 galaxies with $\rho_{h}\left(r_{\min }\right)>4 \rho_{b}\left(r_{\min }\right) \quad$ [5].

\begin{tabular}{|c|c|c|c|c|c|c|c|c|}
\hline \multirow[t]{2}{*}{ Galaxy } & \multicolumn{2}{|c|}{ fermion URTE } & \multicolumn{2}{|c|}{ boson URTE } & \multicolumn{2}{|c|}{ fermion NRTE } & \multicolumn{2}{|c|}{ boson NRTE } \\
\hline & $10^{6} a_{h \mathrm{NR}}^{\prime}$ & $\chi^{2}$ & $10^{6} a_{h \mathrm{NR}}^{\prime}$ & $\chi^{2}$ & $10^{6} a_{h \mathrm{NR}}^{\prime}$ & $\chi^{2}$ & $10^{6} a_{h \mathrm{NR}}^{\prime}$ & $\chi^{2}$ \\
\hline DDO161 & $4.5 \pm 0.2$ & 28.9 & $4.0 \pm 0.2$ & 29.8 & $4.6 \pm 0.2$ & 28.7 & $2.6 \pm 0.2$ & 30.6 \\
\hline F568-1* & $3.3 \pm 0.3$ & 14.2 & $2.9 \pm 0.3$ & 14.1 & $3.3 \pm 0.3$ & 14.3 & $2.0 \pm 0.3$ & 14.4 \\
\hline F574-1 * & $2.8 \pm 0.2$ & 17.2 & $2.4 \pm 0.2$ & 15.4 & $2.8 \pm 0.2$ & 17.8 & $1.6 \pm 0.1$ & 15.4 \\
\hline NGC0024* & $1.8 \pm 0.1$ & 23.2 & $1.5 \pm 0.1$ & 19.7 & $1.8 \pm 0.1$ & 24.7 & $0.9 \pm 0.1$ & 20.7 \\
\hline NGC3109* & $2.9 \pm 0.1$ & 9.2 & $2.8 \pm 0.2$ & 8.5 & $2.9 \pm 0.1$ & 9.5 & $2.8 \pm 0.2$ & 8.3 \\
\hline NGC3972 * & $3.4 \pm 0.3$ & 13.1 & $3.0 \pm 0.3$ & 11.6 & $3.5 \pm 0.3$ & 13.5 & $2.3 \pm 0.3$ & 10.3 \\
\hline NGC4183 * & $3.3 \pm 0.2$ & 42.5 & $2.6 \pm 0.2$ & 43.5 & $3.4 \pm 0.2$ & 42.3 & $1.8 \pm 0.1$ & 44.9 \\
\hline NGC4559 & $3.4 \pm 0.2$ & 33.7 & $2.7 \pm 0.2$ & 30.7 & $3.6 \pm 0.2$ & 34.6 & $2.0 \pm 0.2$ & 28.5 \\
\hline NGC6503 & $2.1 \pm 0.1$ & 49.9 & $1.4 \pm 0.1$ & 51.2 & $2.2 \pm 0.1$ & 50.2 & $0.7 \pm 0.1$ & 55.6 \\
\hline UGC00731* & $2.1 \pm 0.1$ & 11.5 & $1.7 \pm 0.1$ & 9.9 & $2.2 \pm 0.1$ & 12.0 & $1.2 \pm 0.1$ & 8.9 \\
\hline UGC06667 * & $2.5 \pm 0.2$ & 4.5 & $2.2 \pm 0.2$ & 4.8 & $2.6 \pm 0.2$ & 4.5 & $1.7 \pm 0.2$ & 5.6 \\
\hline UGC07125 & $2.9 \pm 0.3$ & 25.1 & $2.3 \pm 0.2$ & 23.5 & $3.1 \pm 0.3$ & 25.7 & $1.5 \pm 0.2$ & 22.1 \\
\hline UGC08490 & $1.3 \pm 0.1$ & 6.6 & $1.0 \pm 0.1$ & 4.3 & $1.4 \pm 0.1$ & 7.7 & $0.7 \pm 0.1$ & 4.5 \\
\hline UGC11914 & $1.3 \pm 0.1$ & 130.5 & $1.1 \pm 0.1$ & 118.8 & $1.4 \pm 0.1$ & 134.6 & $0.95 \pm 0.1$ & 113.5 \\
\hline UGC12632 * & $2.2 \pm 0.1$ & 6.9 & $1.8 \pm 0.1$ & 6.1 & $2.3 \pm 0.1$ & 7.3 & $1.4 \pm 0.1$ & 5.8 \\
\hline Average & 2.65 & $\sum \chi^{2}$ & 2.23 & $\sum \chi^{2}$ & 2.75 & $\sum \chi^{2}$ & 1.61 & $\sum \chi^{2}$ \\
\hline $\begin{array}{c}\text { Standard } \\
\text { dev. }\end{array}$ & 0.85 & 417.0 & 0.80 & 391.9 & 0.86 & 427.3 & 0.64 & 389.1 \\
\hline \multicolumn{9}{|l|}{9 galaxies * } \\
\hline Average & 2.69 & $\sum \chi^{2}$ & 2.34 & $\sum \chi^{2}$ & 2.77 & $\sum \chi^{2}$ & 1.74 & $\sum \chi^{2}$ \\
\hline $\begin{array}{c}\text { Standard } \\
\text { dev. }\end{array}$ & 0.54 & 142.3 & 0.53 & 133.5 & 0.55 & 145.9 & 0.55 & 134.4 \\
\hline
\end{tabular}


Table 3. Measurements of $a_{h \mathrm{NR}}^{\prime}$ with several galaxies, for the case of fermions with URTE. $a_{h \mathrm{NR}}^{\prime}$ has been corrected to $r \rightarrow 0$ by extrapolation. A breakdown of the known uncertainties, at $68 \%$ confidence, is presented. Additional unknown uncertainties include non-steady state galaxies, extraneous features of the rotation curves, phase space dilution, and systematic uncertainties of the measurements.

\begin{tabular}{|c|c|c|c|c|c|c|}
\hline Galaxy & $10^{6} a_{h \mathrm{NR}}^{\prime}$ & stat & $\Upsilon_{*}$ & $k_{h}$ & $v\left(r_{\min }\right)$ & Total \\
\hline & & & 0.2 to 0.5 & 0 to 0.5 & $\pm 1 \sigma$ & \\
\hline DDO161 & 4.52 & \pm 0.19 & \pm 0.20 & \pm 0.74 & \pm 0.033 & \pm 0.79 \\
\hline F568-1 & 3.25 & \pm 0.30 & \pm 0.08 & \pm 0.53 & \pm 0.001 & \pm 0.62 \\
\hline F574-1 & 2.77 & \pm 0.15 & \pm 0.03 & \pm 0.46 & \pm 0.001 & \pm 0.48 \\
\hline NGC0024 & 1.78 & \pm 0.06 & \pm 0.10 & \pm 0.29 & \pm 0.001 & \pm 0.31 \\
\hline UGC11914 & 1.25 & \pm 0.05 & \pm 0.63 & \pm 0.29 & \pm 0.035 & \pm 0.70 \\
\hline UGC12632 & 2.21 & \pm 0.13 & \pm 0.04 & \pm 0.39 & \pm 0.000 & \pm 0.41 \\
\hline
\end{tabular}

Table 4. Summary of measurements of the adiabatic invariant $v_{h r m s}(1)$ defined in (1), the expansion parameter at which dark matter particles become non-relativistic $a_{h \mathrm{NR}}^{\prime} \equiv v_{h \mathrm{rms}}(1) / c$, the cut-off wavenumber of warm dark matter $k_{\mathrm{fs}}$ defined in (7), the free-streaming galaxy transition mass $M_{\mathrm{fs}} \equiv 4 \pi\left(1.555 / k_{\mathrm{fs}}\right)^{3} \Omega_{m} \rho_{\text {crit }} / 3$, and the mass $m_{h}$ of dark matter particles, for four dark matter scenarios with zero chemical potential. Shown are total uncertainties with $68 \%$ confidence. Ranges from "No freeze-in/-out" are hard limits.

\begin{tabular}{|c|c|c|c|c|c|}
\hline Observable & $v_{h \mathrm{mms}}(1)$ & $10^{6} a_{h \mathrm{NR}}^{\prime}$ & $k_{\mathrm{fs}}$ & $\log _{10}\left(M_{\mathrm{fs}} / M_{\odot}\right)$ & $m_{h}$ \\
\hline & {$[\mathrm{km} / \mathrm{s}]$} & & {$\left[\mathrm{Mpc}^{-1}\right]$} & & {$[\mathrm{eV}]$} \\
\hline \multicolumn{6}{|l|}{ Fermions URTE } \\
\hline Spiral galaxies & $0.79 \pm 0.26$ & $2.65 \pm 0.85$ & $0.25_{-0.05}^{+0.10}$ & $13.5 \pm 0.4$ & $107_{-20}^{+36}$ \\
\hline No freeze-in/-out & 2.00 to 0.75 & 6.66 to 2.50 & 0.12 to 0.26 & 14.5 to 13.5 & 54 to 112 \\
\hline$M_{s}$ distribution & & & $0.90_{-0.34}^{+0.44}$ & $11.9 \pm 0.6$ & \\
\hline \multicolumn{6}{|l|}{ Bosons URTE } \\
\hline Spiral galaxies & $0.67 \pm 0.24$ & $2.23 \pm 0.80$ & $0.37_{-0.08}^{+0.17}$ & $13.0 \pm 0.4$ & $124_{-25}^{+50}$ \\
\hline No freeze-in/-out & 1.19 to 0.45 & 3.97 to 1.49 & 0.23 to 0.52 & 13.6 to 12.6 & 81 to 168 \\
\hline$M_{s}$ distribution & & & $0.90_{-0.40}^{+0.44}$ & $11.9 \pm 0.7$ & \\
\hline \multicolumn{6}{|l|}{ Fermions NRTE } \\
\hline Spiral galaxies & $0.82 \pm 0.26$ & $2.75 \pm 0.86$ & $0.21_{-0.04}^{+0.07}$ & $13.8 \pm 0.4$ & $74_{-14}^{+24}$ \\
\hline No freeze-in/-out & 1.04 to 0.39 & 3.46 to 1.30 & 0.17 to 0.38 & 14.0 to 13.0 & 62 to 130 \\
\hline$M_{s}$ distribution & & & $0.90_{-0.34}^{+0.44}$ & $11.9 \pm 0.6$ & \\
\hline \multicolumn{6}{|l|}{ Bosons NRTE } \\
\hline Spiral galaxies & $0.48 \pm 0.19$ & $1.61 \pm 0.64$ & $0.92_{-0.24}^{+0.54}$ & $11.8 \pm 0.5$ & $73_{-17}^{+33}$ \\
\hline No freeze-in/-out & 0.36 to 0.14 & 1.21 to 0.45 & 1.19 to 3.00 & 11.5 to 10.3 & 90 to 188 \\
\hline$M_{s}$ distribution & & & $0.90_{-0.40}^{+0.44}$ & $11.9 \pm 0.7$ & \\
\hline
\end{tabular}




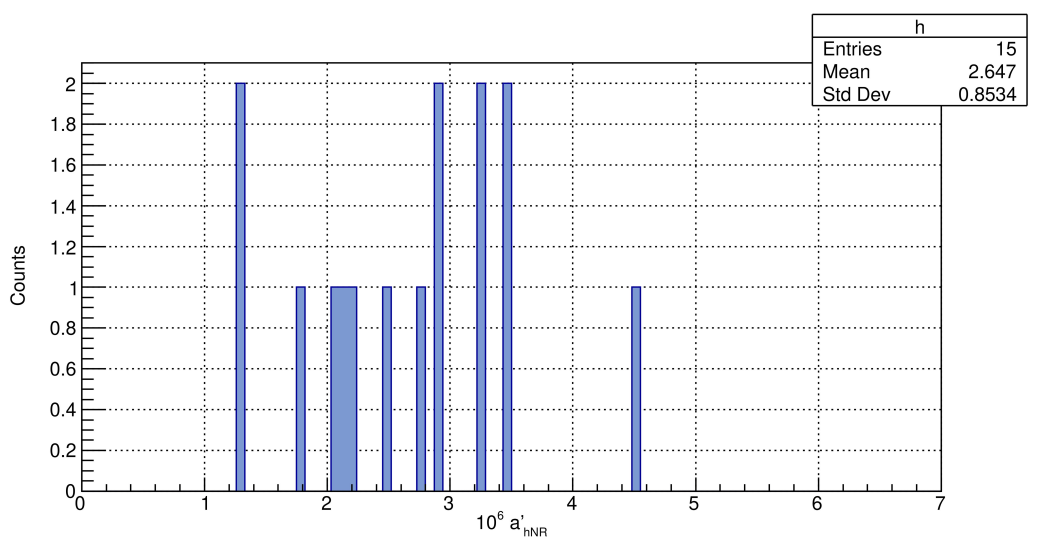

Figure 6. Distribution of the measured $a_{h \mathrm{NR}}^{\prime}$ for the case of fermions with URTE and $\mu^{\prime}=0$ from Table 2 .

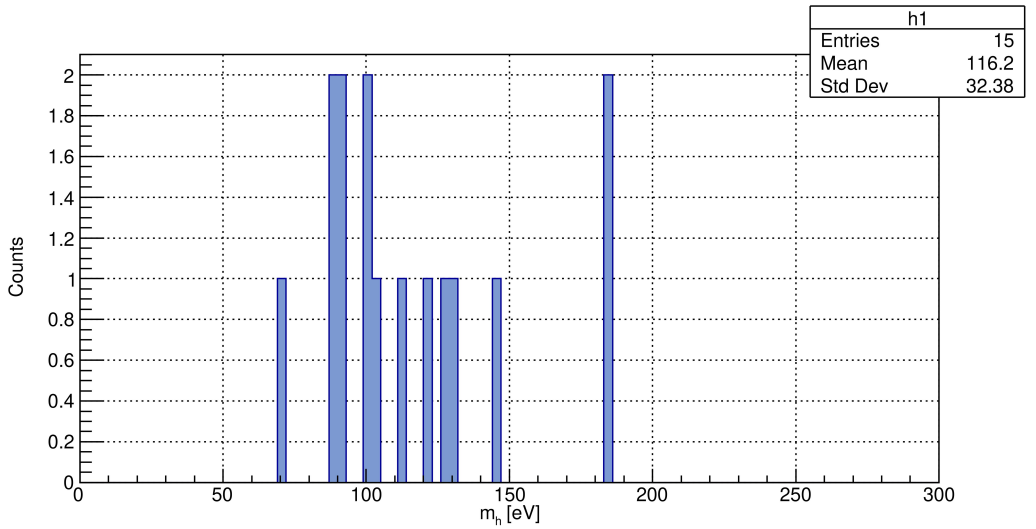

Figure 7. Distribution of measured dark matter masses for the case of fermions with URTE and $\mu^{\prime}=0$.

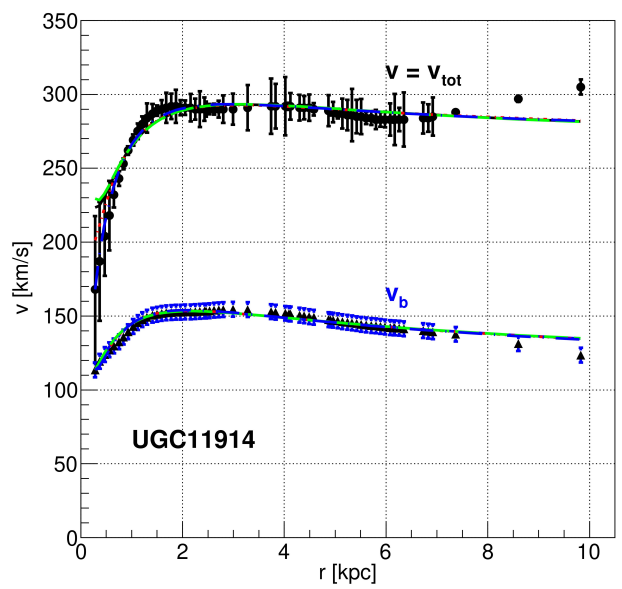

Figure 8. Rotation velocities $v(r) \equiv v_{\text {tot }}(r)$ of galaxy UGC11914 as a function of the distance $r$ from the center of the galaxy, taken from the SPARC catalog [3]. $v_{b}$ is the contribution from baryons. The curves are fitted numerical integrations for fermions with URTE (black continuous lines), bosons with URTE (red dot-short dash), fermions with NRTE (green long dash), and bosons with NRTE (blue dot-long dash). The numerical integrations start with $\mu^{\prime}=0$ at $r \rightarrow 0$. The $\chi^{2}$ 's are presented in Table 2. 
Note, in Table 4, that we have measured dark matter particle masses of order $100 \mathrm{eV}$ (with (15), (17), (23), or (25)). For fermion dark matter, these measurements are in disagreement with limits obtained from dwarf spheroidal (dSph) "satellites" of the Milky Way, assuming that they are dominated by dark matter, i.e. $m_{h}>410 \mathrm{eV}$ from the Pauli exclusion principle, and even more stringent limits with additional assumptions, e.g. the Tremaine-Gunn limit [11]. However, recent studies suggest that dwarf spheroidals are not satellites of the Milky Way, they are on their first entry to the Galaxy, and contain negligible amounts of dark matter [12] [13] [14] [15]! This disagreement needs to be resolved if dark matter is composed of fermions. For bosons, there is no issue.

We have made several measurements of $a_{h \mathrm{NR}}^{\prime}: a_{h \mathrm{NR}}^{\prime}=(4.17 \pm 2.52) \times 10^{-6}$ with ten galaxies in the THINGS sample [2] [4], and $a_{h \mathrm{NR}}^{\prime}=(2.54 \pm 0.97) \times 10^{-6}$ with fourty six different galaxies in the SPARC sample [3] [5], to be compared with the measurements in Table 2.

\section{Free-Streaming}

Free-streaming is important at expansion parameters of order $a_{h \mathrm{NR}}^{\prime}$, long after dark matter has decoupled, see Section 5. We therefore consider collision-less dark matter with zero chemical potential. A density perturbation corresponds to a temperature fluctuation, i.e. to a change in the momentum distribution of the particles, see Appendix A and Appendix B. The comoving free-streaming distance of a dark matter particle of momentum $p=p_{1} / a$ is

$$
\begin{aligned}
d_{\mathrm{fs}}\left(p_{1}\right) & =\int_{0} \frac{v_{h}(a) \cdot \mathrm{d} t}{a}=\int_{0} \frac{c}{\sqrt{1+\left(a /\left[p_{1} /\left(m_{h} c\right)\right]\right)^{2}}} \frac{\mathrm{d} t}{a}, \\
& =\int_{0}^{\mathrm{dec}} \frac{c \cdot \mathrm{d} a}{\sqrt{1+\left(a /\left[p_{1} /\left(m_{h} c\right)\right]\right)^{2}} H_{0} a^{2} \sqrt{\Omega_{r} a^{-4}+\Omega_{m} a^{-3}}} .
\end{aligned}
$$

(We arbitrarily stop the integral at decoupling as further contributions are of order 5\%.) Let $P(k)$ be the power spectrum of linear density perturbations in the cold dark matter $\Lambda \mathrm{CDM}$ model. $k$ is the comoving wavenumber. The power spectrum for warm dark matter is $P(k) \tau^{2}\left(k / k_{\mathrm{fs}}\right)$, where $\tau^{2}\left(k / k_{\mathrm{fs}}\right)$ is a cut-off factor.

Let $\delta_{h}(\mathbf{x}) \equiv[\rho(\mathbf{x})-\bar{\rho}] / \bar{\rho}$ be the normalized dark matter density perturbation, and $a_{h}(\mathbf{k})$ its Fourier transform. We partition $\delta_{h}(\mathbf{x})$ into parts that free-stream into different elements of solid angle $\mathrm{d} \Omega$. Due to free-streaming of dark matter, the corresponding part of $a_{h}(\mathbf{k})$ becomes multiplied by $\exp \left[i k \cos \theta d_{\mathrm{fs}}\left(p_{1}\right)\right]$, where $\theta$ is the angle between $\mathbf{k}$ and $\mathbf{d}_{\text {fs }}\left(p_{1}\right)$. This factor needs to be averaged over $\Omega$, and over the comoving momentum $p_{1}$ from 0 to $\infty$. The average of the imaginary part is zero, so we need only average $\cos \left(k \cos \theta d_{\mathrm{fs}}\left(p_{1}\right)\right)$. The average of this term over $\Omega$ obtains $\sin \left[k d_{\mathrm{fs}}\left(p_{1}\right)\right] /\left[k d_{\mathrm{fs}}\left(p_{1}\right)\right]$. We take the $p_{1}$ average only over the perturbation of the momentum distribution of the dark matter particles (as other free-streaming cancels by detailed balance). The results are presented in Figure 9. This figure has $\mu^{\prime}=0$ for fermions. $\mu^{\prime}=0$ is singular for 
bosons, so for this figure we take $\mu^{\prime}=-0.01$. For fermions with URTE or NRTE, the cut-off factor $\tau^{2}\left(k / k_{\mathrm{fs}}\right)$ is well approximated by

$$
\tau^{2}\left(k / k_{\mathrm{fs}}\right)=\exp \left(-k^{2} / k_{\mathrm{fs}}^{2}\right) .
$$

We use $\tau^{2}(1) \equiv \mathrm{e}^{-1}$ as our definition of the free-streaming cut-off wavenumber $k_{\mathrm{fs}}$. This precise definition supersedes the qualitative definition of the cut-off wavenumber in previous publications [7]. Equation (7) is consistent with the definition of $k_{\mathrm{fs}}$ used in Figures 10-15 [8]. For bosons with URTE or NRTE, there is a tail at large $k$ due to the excess of low momentum dark matter particles in the limit $\mu^{\prime} \rightarrow-0$. This tail depends on $\mu^{\prime}$, and may have cosmological consequences.

The approximation $\tau^{2}\left(k / k_{\mathrm{fs}}\right) \approx \exp \left(-k^{2} / k_{\mathrm{fs}}^{2}\right)$ is convenient: it allows the definition of the "free-streaming transition mass"

$$
M_{\mathrm{fs}}=\frac{4}{3} \pi\left(\frac{1.555}{k_{\mathrm{fs}}}\right)^{3} \Omega_{m} \rho_{\text {crit }} .
$$

The factor 1.555 comes from the Fourier transform of a 3-dimensional Gaussian. Free-streaming affects the distribution of halo masses with $M<M_{\mathrm{fs}}$. The cut-off wavenumbers $k_{\mathrm{fs}}$, and the free-streaming masses $M_{\mathrm{fs}}$, corresponding to the measured values of $a_{h \mathrm{NR}}^{\prime}$, are summarized in Table 4 . We verify that perturbations with $k<k_{\mathrm{fs}}$ grow due to gravitational instability, i.e. $k_{\mathrm{fs}}<k_{J}$, where $k_{J}$ is the Jeans wavenumber for collision-less dark matter [9] [16]. Note that for $k \approx k_{\mathrm{fs}}$ it is the slower particles that survive free-streaming.

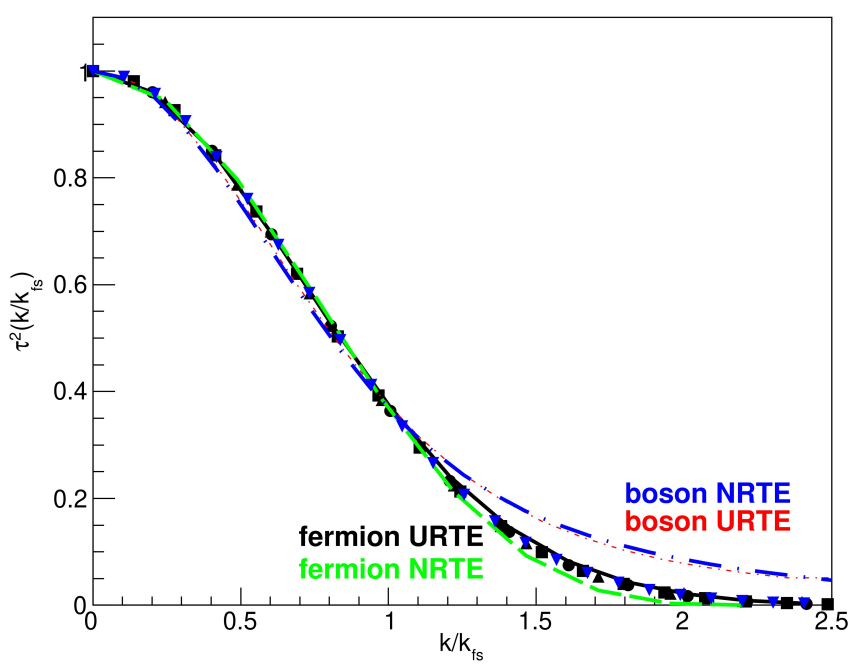

Figure 9. Cut-off factor $\tau^{2}\left(k / k_{\mathrm{fs}}\right)$ for fermions with URTE (black continuous line), bosons with URTE (red dot-short dash), fermions with NRTE (green long dash), and bosons with NRTE (blue dot-long dash). Markers correspond to the approximation $\exp \left(-k^{2} / k_{\mathrm{fs}}^{2}\right) . \quad \mu^{\prime} \equiv \mu^{\prime}(0)=0$ for fermions, $\mu^{\prime}=-0.01$ for bosons. The normalization is $\tau^{2}(0)=1$, and the definition of $k_{\mathrm{fs}}, \tau^{2}(1)=\mathrm{e}^{-1}$. The long tails for bosons depend on $\mu^{\prime}$. For NRTE bosons, $\tau^{2}(2)=0.03,0.09,0.15,0.20,0.24$ for $\mu^{\prime}=-0.1,-0.01,-0.001,-0.0001,-0.00001$, respectively. 


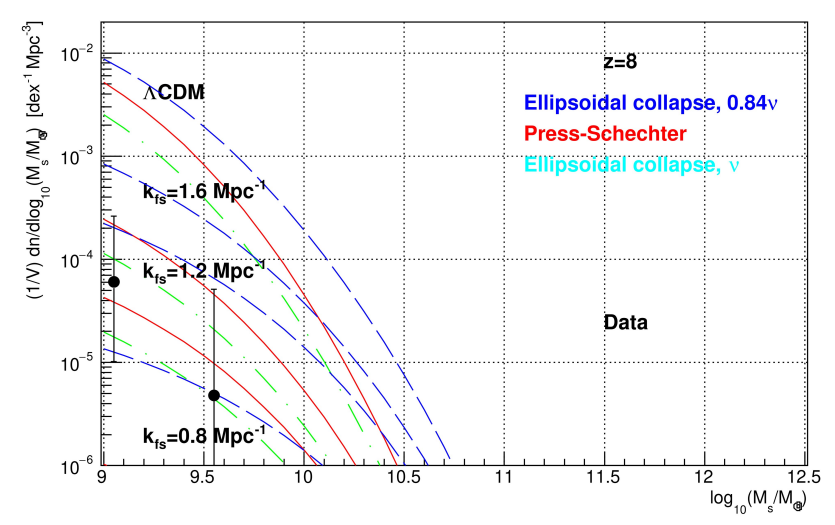

Figure 10. Calculated stellar mass functions with the Press-Schechter [17], Ellipsoidal Collapse with $\tilde{v}=v$, and Ellipsoidal Collapse with $\tilde{v}=0.84 v$ [18] [19], approximations, for $\Lambda \mathrm{CDM}$, and $\Lambda \mathrm{WDM}$ with $k_{\mathrm{fs}}=1.6,1.2$ and $0.8 \mathrm{Mpc}^{-1}$, at redshift $z=8$, compared with observations [20] [21] [22]. $\log _{10}(M)=\log _{10}\left(M_{s}\right)+1.5$.

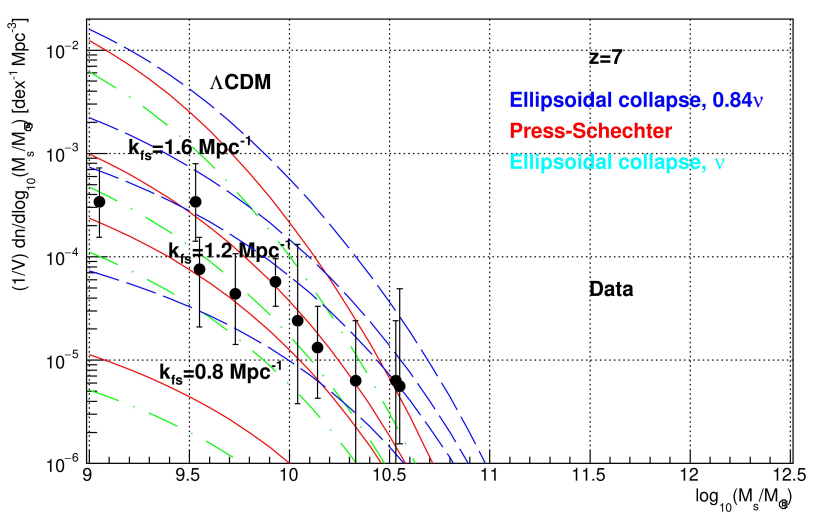

Figure 11. Calculated stellar mass functions with the Press-Schechter, Ellipsoidal Collapse with $\tilde{v}=v$, and Ellipsoidal Collapse with $\tilde{v}=0.84 v$, approximations, for $\Lambda \mathrm{CDM}$, and $\Lambda \mathrm{WDM}$ with $k_{\mathrm{fs}}=1.6,1.2$ and $0.8 \mathrm{Mpc}^{-1}$, at redshift $z=7$, compared with observations [20] [21] [22]. $\log _{10}(M)=\log _{10}\left(M_{s}\right)+1.5$.

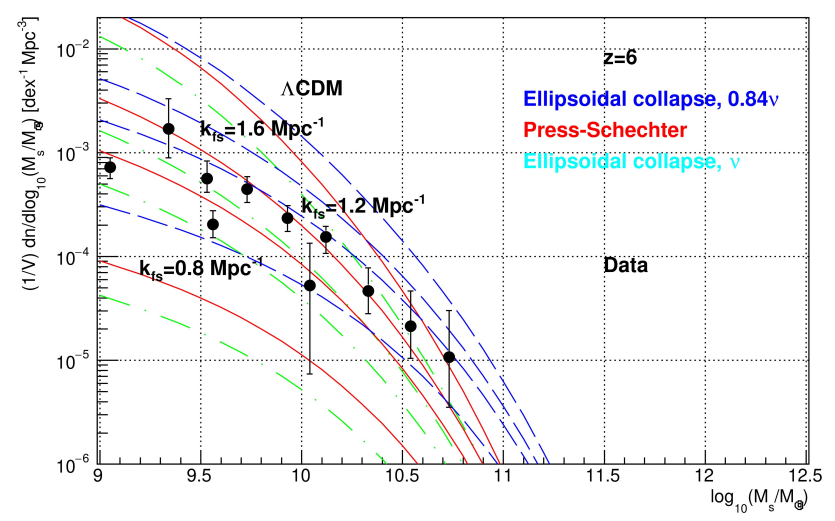

Figure 12. Calculated stellar mass functions with the Press-Schechter, Ellipsoidal Collapse with $\tilde{v}=v$, and Ellipsoidal Collapse with $\tilde{v}=0.84 v$, approximations, for $\Lambda \mathrm{CDM}$, and $\Lambda \mathrm{WDM}$ with $k_{\mathrm{fs}}=1.6,1.2$ and $0.8 \mathrm{Mpc}^{-1}$, at redshift $z=6$, compared with observations [20] [21] [22]. $\log _{10}(M)=\log _{10}\left(M_{s}\right)+1.5$. 


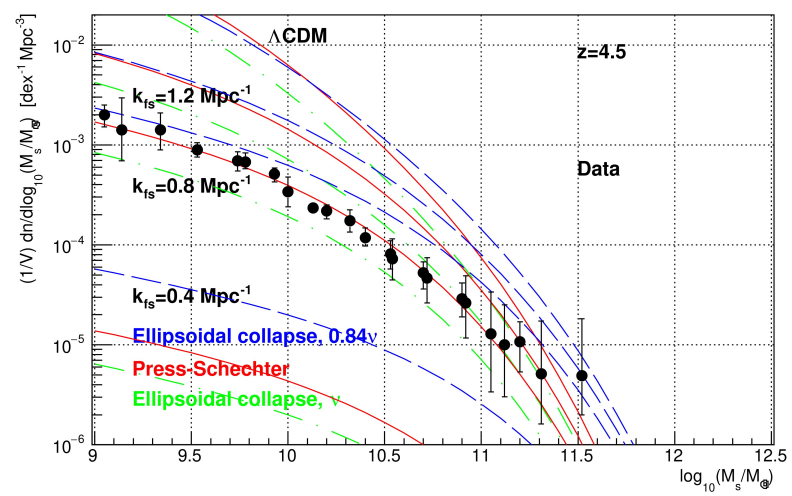

Figure 13. Calculated stellar mass functions with the Press-Schechter, Ellipsoidal Collapse with $\tilde{v}=v$, and Ellipsoidal Collapse with $\tilde{v}=0.84 v$ approximations, for $\Lambda \mathrm{CDM}$, and $\Lambda \mathrm{WDM}$ with $k_{\mathrm{fs}}=1.2,0.8$ and $0.4 \mathrm{Mpc}^{-1}$, at redshift $z=4.5$, compared with observations [20] [21] [22] [23]. $\log _{10}(M)=\log _{10}\left(M_{s}\right)+1.5$.

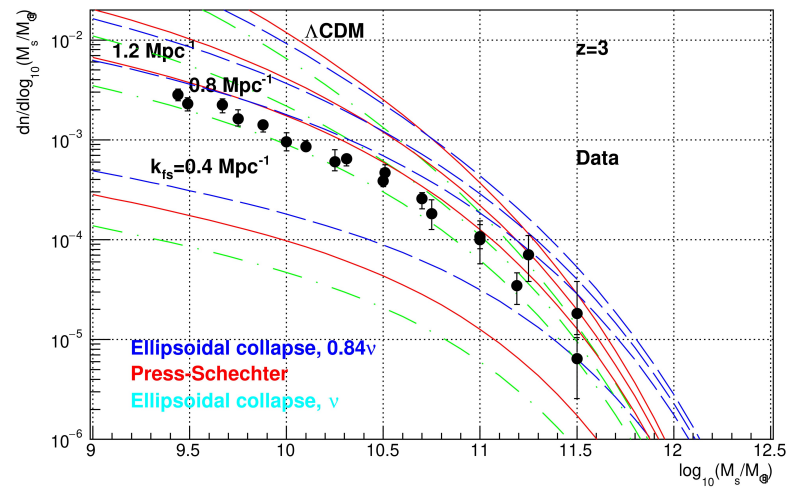

Figure 14. Calculated stellar mass functions with the Press-Schechter, Ellipsoidal Collapse with $\tilde{v}=v$, and Ellipsoidal Collapse with $\tilde{v}=0.84 v$ approximations, for $\Lambda$ CDM, and $\Lambda \mathrm{WDM}$ with $k_{\mathrm{fs}}=1.2,0.8$ and $0.4 \mathrm{Mpc}^{-1}$, at redshift $z=3$, compared with observations [20] [21] [22] [23]. $\log _{10}(M)=\log _{10}\left(M_{s}\right)+1.5$.

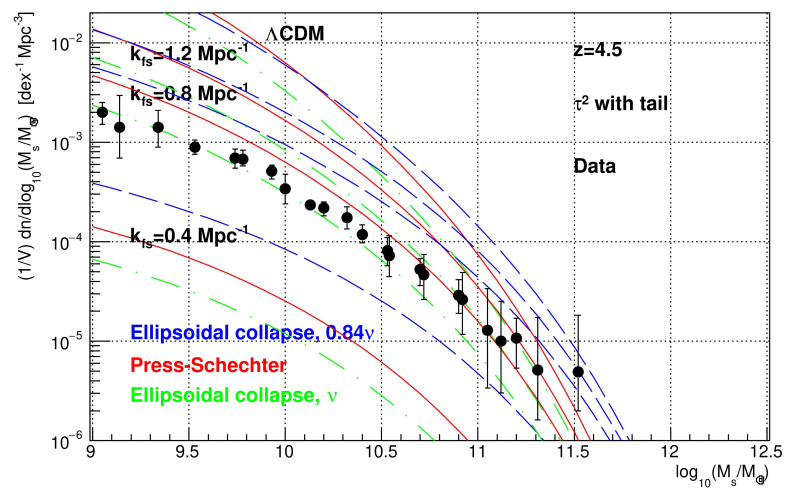

Figure 15. Same as Figure 13, except that the cut-off factor has a "tail" that applies to boson dark matter, see (9). $z=4.5$.

\section{No Freeze-In and No Freeze-Out}

We assume that dark matter is in thermal and diffusive equilibrium with the 
standard model sector in the early universe, and decouples (from the standard model sector, and from self-annihilation) while still ultra-relativistic. As the universe expands and cools, standard model particles and anti-particles become non-relativistic and annihilate, heating the standard model sector, without heating dark matter if it has already decoupled. Let $T_{h} / T$ be the ratio of the dark matter-to-photon temperatures after $\mathrm{e}^{+} \mathrm{e}^{-}$annihilation (and, in the case NRTE, before dark matter becomes non-relativistic). If dark matter decouples at temperatures $T>m_{t}$, then $T_{h} / T=[8 \times 43 /(427 \times 22)]^{1 / 3}=0.332$. If dark matter decouples in the temperature range $T_{C}<T<m_{s}$, then $T_{h} / T=[8 \times 43 /(205 \times 22)]^{1 / 3}=0.424$. These numbers can be found in Section 22.3.2 of [1]. $T_{C} \approx 0.2 \mathrm{GeV}$ corresponds to the confinement-deconfinement transition. If dark matter decouples at temperatures $T<T_{C}$ there is disagreement with Big Bang Nucleosynthesis (BBN). Thus, we have the hard limits $0.332 \leq T_{h} / T \leq 0.424$. With the expressions in Appendix A and Appendix B, and the calculations of free-streaming, we fill the rows "No freeze-in/-out" in Table 4.

From Table 4 we conclude that all four extreme scenarios studied in this article, namely, fermions with URTE, bosons with URTE, fermions with NRTE, and bosons with NRTE, with $\mu^{\prime}=0$, and with $N_{f}=2$ or $N_{b}=1$, are consistent with thermal equilibrium of dark matter with the standard model sector in the early universe. This consistency is non-trivial as it depends on the measurement of $a_{h \mathrm{NR}}^{\prime}$ with each of 56 spiral galaxies [4] [5] [6], and the measurement of the cosmic microwave background temperature $T_{0}$.

\section{Galaxy Stellar Mass Distributions}

Figures 10-13 compare galaxy stellar mass distribution predictions with observations. These figures were taken from Reference [8] with several changes: 1) In [8] all theoretical curves plot $(1 / V) \mathrm{d} n / \mathrm{d} \ln \left(M / M_{\odot}\right):$ I missed a factor $\ln (10)$ to convert to $(1 / V) \mathrm{d} n / \mathrm{d}_{\log _{10}}\left(M / M_{\odot}\right)$ ! This error has now been corrected. 2) The cold and warm dark matter models coincide for halo masses $M>M_{\mathrm{fs}}$, and differ for $M<M_{\mathrm{fs}}$. Therefore, to measure the cut-off wavenumber $k_{\mathrm{fs}}$, we first adjust the relation between the halo mass $M$ and the stellar mass $M_{s}$ to obtain agreement for $M>M_{\mathrm{fs}}$, and obtain $\log _{10}\left(M_{s} / M\right)=-1.5$, consistent with Figure 9 of Reference [20].3) We apply the cut-off factor (7) without the "tail", and 4) Update the cosmological parameters to [1]. From Figures 10-13, and the Sheth-Tormen ellipsoidal collapse prediction with $\tilde{v}=0.84 v$ [18] [19], we obtain $k_{\mathrm{fs}}=0.90_{-0.34}^{+0.44} \mathrm{Mpc}^{-1}$ as in Reference [8]. For completeness, we include Figure 14 for $z=3$, but do not use it because the mass fraction locked up in halos of mass greater than $M, F(M, z)$, exceeds 0.01 at $M=10^{9} M_{\odot}$, and saturation sets in.

We repeat Figure 13 with the cut-off factor with a "tail" that corresponds to bosons with $\mu^{\prime} \approx-0.01$ :

$$
\tau^{2}\left(k / k_{\mathrm{fs}}\right)= \begin{cases}\exp \left(-k^{2} / k_{\mathrm{fs}}^{2}\right) & \text { for } k<k_{\mathrm{fs}} \\ \exp \left(-k / k_{\mathrm{fs}}\right) & \text { for } k>k_{\mathrm{fs}}\end{cases}
$$


and obtain Figure 15. Since bosons may have $\tau^{2}\left(k / k_{\mathrm{fs}}\right)$ with a "tail", we estimate $k_{\mathrm{fs}}=0.90_{-0.40}^{+0.44} \mathrm{Mpc}^{-1}$ for bosons.

These results for $k_{\mathrm{fs}}$ are in disagreement with studies of the Lyman- $\alpha$ forest. The Lyman- $\alpha$ forest allows measurements of the neutral hydrogen density profile along the line of sight to far away quasars (at redshifts $z \approx 5.5$ ). From the analysis of these density profiles, with model dependent simulations of the inter-galactic medium (including the highly ionized hydrogen), the cut-off wavenumber $k_{\mathrm{fs}}$ is excluded in the range from $\approx 0.4 \mathrm{Mpc}^{-1}$ to $\approx 27 \mathrm{Mpc}^{-1}$ [24]. So, these two analysis, based on very different data sets, are in tension. For boson dark matter, the long free-streaming "tail" mitigates the tension. This discrepancy needs to be resolved.

\section{Conclusions}

From this and previous [4]-[9] studies we arrive at the following conclusions:

1) Each spiral galaxy allows a measurement of the adiabatic invariant $a_{h \mathrm{NR}}^{\prime} \equiv v_{h \mathrm{rms}}(1) / c$. We find that $a_{h \mathrm{NR}}^{\prime}$ has the same value in the core of all measured relaxed steady state spiral galaxies (within statistical and systematic uncertainties). Therefore, we interpret $a_{h \mathrm{NR}}^{\prime}$ to be of cosmological origin: it is the expansion parameter at which dark matter particles become non-relativistic. $a_{h \mathrm{NR}}^{\prime}$ determines the ratio of dark matter temperature to mass $T_{h}(a) / m_{h}$ in the early universe. To obtain $T_{h}(a)$ and $m_{h}$ separately, we need one more constraint, i.e. the value of $\mu^{\prime} \equiv \mu /\left(k T_{h}\right)$, where $\mu$ is the chemical potential.

2) The present dark matter density of the universe $\Omega_{c} \rho_{\text {crit }}$ determines the dark matter particle mass $m_{h}$ as a function of $a_{h \mathrm{NR}}^{\prime}$ and $\mu^{\prime}$, see Appendix A and Appendix B. Therefore, if $a_{h \mathrm{NR}}^{\prime}$ has the same value in the core of all relaxed steady state spiral galaxies, we can expect the same for $\mu^{\prime}$, so $\mu^{\prime}$ may be of cosmological origin.

3) The measured value of $v_{h r m s}(1)$ corresponds to thermal equilibrium of dark matter with the standard model sector in the early universe, with no freeze-in and no freeze-out, if $\mu^{\prime}=0$ (see Section 5, and (16), (18), (24), and (26)). Thus, we have obtained either a coincidence, or strong evidence that $\mu^{\prime}=0$. Therefore, we assume $\mu^{\prime}=0$, and arrive at the four dark matter scenarios studied in this article.

4) With $\mu^{\prime}=0$, each spiral galaxy allows an independent measurement of the dark particle mass $m_{h}$. The results are consistent within uncertainties.

5) The dark particle masses listed in Table 4 were obtained from data, without reference to any particular extension of the standard model. These measurements are in tension with some limits. A comment on the Tremaine-Gunn limit is made in Section 3, and a comment on the Lyman- $\alpha$ forest limit is included in Section 6. Comments on limits from strong gravitational lensing, and from the UV luminosity function are addressed in [9]. These tensions need to be resolved. Nature will have the last word.

6) From the measured values of $a_{h \mathrm{NR}}^{\prime}$, and $\mu^{\prime}=0$, we calculate the warm 
dark matter cut-off wavenumbers $k_{\mathrm{fs}}$ due to free-streaming, see Table 4 .

7) Galaxy stellar mass distributions, presented in Figures 10-15, show convincing evidence that dark matter is warm with a cut-off wavenumber $k_{\mathrm{fs}}=0.90_{-0.34}^{+0.44}$ for fermions, or $k_{\mathrm{fs}}=0.90_{-0.40}^{+0.44}$ for bosons (the difference is due to the excess of low momentum bosons expected for $\mu^{\prime} \rightarrow-0$, which produces a "tail" in the cut-off factor $\tau^{2}\left(k / k_{\mathrm{fs}}\right)$, see Figure 9).

8) Fits to spiral galaxy rotation curves generally favor boson dark matter, typically as shown in Figure 1 and Figure 5, but the difference in $\chi^{2}$ for individual galaxies is not statistically significant, see Table 2 . An exception is galaxy UGC11914 (see Figure 8), but this case needs to be taken with caution because the core of UGC11914 is not dominated by dark matter. Among the galaxies with the core dominated by dark matter, the one with the largest $\chi^{2}$ difference between fermions and bosons is NGC0024 with $\Delta \chi^{2}=4$. From the sums of $\chi^{2}$ 's of galaxies with the core dominated by dark matter, we obtain $\sum \Delta \chi^{2}=8.8$ for URTE, and 11.5 for NRTE, corresponding to a discrimination of $3.0 \sigma$ or $3.4 \sigma$, see Table 2 .

9) From Table 4 we observe that fits to spiral galaxy rotation curves obtain agreement with the assumption of no freeze-in and no freeze-out, for each of the four scenarios with $\mu^{\prime}=0$ studied in this article.

10) In Table 4 we see that the cut-off wavenumber $k_{\mathrm{fs}}$, measured with the galaxy stellar mass distributions, is in some tension with fermion dark matter. In fact, from Figures $10-15$ it is difficult to see how $k_{\mathrm{fs}}$ can reach 0.38 or 0.26 $\mathrm{Mpc}^{-1}$ as required by fermions, see Table 4 .

11) To summarize, among the four well motivated dark matter scenarios studied in this article, measurements show evidence for boson dark matter with a significance of $3.5 \sigma$, see Table 2 and Table 4, and obtain no significant discrimination between URTE and NRTE.

\section{Conflicts of Interest}

The author declares no conflicts of interest regarding the publication of this paper.

\section{References}

[1] Zyla, P.A., et al. (Particle Data Group) (2020) 2020 Review of Particle Physics. Prog. Theor. Exp. Phys., 2020, 083C01. https://pdglive.lbl.gov/Viewer.action

[2] de Blok, W.J.G., et al. (2008) High-Resolution Rotation Curves and Galaxy Mass Models from THINGS. The Astronomical Journal, 136, 2648-2719. https://doi.org/10.1088/0004-6256/136/6/2648

[3] Lelli, F., McGaugh, S.S. and Schombert (2016) SPARC: Mass Models for 175 Disk Galaxies with Spitzer Photometry and Accurate Rotation Curves. The Astronomical Journal, 152, 157. https://doi.org/10.3847/0004-6256/152/6/157

[4] Hoeneisen, B. (2019) A Study of Dark Matter with Spiral Galaxy Rotation Curves. International Journal of Astronomy and Astrophysics, 9, 71-96. https://doi.org/10.4236/ijaa.2019.92007 
[5] Hoeneisen, B. (2019) The Adiabatic Invariant of Dark Matter in Spiral Galaxies. International Journal of Astronomy and Astrophysics, 9, 355-367.

[6] Hoeneisen, B. (2019) A Study of Dark Matter with Spiral Galaxy Rotation Curves. Part II. International Journal of Astronomy and Astrophysics, 9, 133-141. https://doi.org/10.4236/ijaa.2019.92010

[7] Hoeneisen, B. (2019) Simulations and Measurements of Warm Dark Matter Free-Streaming and Mass. International Journal of Astronomy and Astrophysics, 9 , 368-392. https://doi.org/10.4236/ijaa.2019.94026

[8] Hoeneisen, B. (2020) Cold or Warm Dark Matter? A Study of Galaxy Stellar Mass Distributions. International Journal of Astronomy and Astrophysics, 10, 57-70. https://doi.org/10.4236/ijaa.2020.102005

[9] Hoeneisen, B. (2020) What Is Dark Matter Made of? Proceedings of the 3rd World Summit on Exploring the Dark Side of the Universe, Guadeloupe Islands, 9-13 March 2020. https://indico.cern.ch/event/801461/overview

[10] Feynman, R.P., Leighton, R.B. and Sands, M. (1963) The Feynman Lectures on Physics. Addison-Wesley Publishing Company, Boston.

[11] Boyarsky, A., Ruchayskiyc, O. and Iakubovskyi, D. (2009) A Lower Bound on the Mass of Dark Matter Particles. Journal of Cosmology and Astroparticle Physics, 3, 005. https://arxiv.org/abs/0808.3902 https://doi.org/10.1088/1475-7516/2009/03/005

[12] Hammer, F., Yang, Y.B., Arenou, F., Puech, M., Flores, H. and Babusiaux, C. (2019) On the Absence of Dark Matter in Dwarf Galaxies Surrounding the Milky Way. ApJ, 883, 171. https://doi.org/10.3847/1538-4357/ab36b6

[13] Hammer, F., Yang Y., Arenou F., Wang J., Li H., Bonifacio P. and Babusiaux, C. (2020) Orbital Evidences for Dark-Matter-Free Milky Way Dwarf Spheroidal Galaxies. The Astrophysical Journal, 892, 3. https://doi.org/10.3847/1538-4357/ab77be

[14] Yang, Y., Hammer, F., Fouquet, S., Flores, H., Puech, M., Pawlowski, M.S. and Kroupa, P. (2014) Reproducing Properties of MW dSphs as Descendants of DM-Free TDGs. MNRAS, 442, 2419-2433. https://doi.org/10.1093/mnras/stu931

[15] Hammer, F., Yang, Y.B., Arenou, F., Babusiaux, C., Puech, M. and Flores, H. (2018) Galactic Forces Rule the Dynamics of Milky Way Dwarf Galaxies. The Astrophysical Journal, 860, 76. https://doi.org/10.3847/1538-4357/aac3da

[16] Boyanovsky, D., de Vega, H.J. and Sanchez, N.G. (2008) The Dark Matter Transfer Function: Free Streaming, Particle Statistics and Memory of Gravitational Clustering. Physical Review D, 78, Article ID: 063546.

https://doi.org/10.1103/PhysRevD.78.063546

[17] Press, W.H. and Schechter, P. (1974) Formation of Galaxies and Clusters of Galaxies by Self-Similar Gravitational Condensation. The Astrophysical Journal, 187, 425-438. https://doi.org/10.1086/152650

[18] Sheth, R.K. and Tormen, G. (1999) Large-Scale Bias and the Peak Background Split. Monthly Notices of the Royal Astronomical Society, 308, 119-126.

https://doi.org/10.1046/j.1365-8711.1999.02692.x

[19] Sheth, R.K., Mo, H.J. and Tormen, G. (2001) Ellipsoidal Collapse and an Improved Model for the Number and Spatial Distribution of Dark Matter Haloes. Monthly Notices of the Royal Astronomical Society, 323, 1-12. https://doi.org/10.1046/j.1365-8711.2001.04006.x

[20] Lapi, A., et al. (2017) Stellar Mass Function of Active and Quiescent Galaxies via the Continuity Equation. The Astrophysical Journal, 847, 13. https://arxiv.org/pdf/1708.07643.pdf 
https://doi.org/10.3847/1538-4357/aa88c9

[21] Song, M., Finkelstein, S.L., Ashby, M.L.N., et al. (2016) The Evolution of the Galaxy Stellar Mass Function at Z $=4-8$ : A Steepening Low-Mass-End Slope with Increasing Redshift. The Astrophysical Journal, 825, 5.

https://doi.org/10.3847/0004-637X/825/1/5

[22] Grazian, A., Fontana, A., Santini, P., et al. (2015) The Galaxy Stellar Mass Function at $3.5 \leq \mathrm{Z} \leq 7.5$ in the CANDELS/UDS, GOODS-South, and HUDF Fields. $A s$ tronomy and Astrophysics, 575, A96. https://doi.org/10.1051/0004-6361/201424750

[23] Davidzon, I., Ilbert, O., Laigle, C., et al. (2017) The COSMOS2015 Galaxy Stellar Mass Function: 13 Billion Years of Stellar Mass Assembly in 10 Snapshots. $A s$ tronomy and Astrophysics, 605, A70. https://doi.org/10.1051/0004-6361/201730419

[24] Baur, J., et al. (2016) Lyman-Alpha Forests Cool Warm Dark Matter. https://arxiv.org/pdf/1512.01981.pdf 


\section{Appendix A: Non-Relativistic Dark Matter in Non-Relativistic Thermal Equilibrium (NRTE)}

In this Section we consider non-relativistic dark matter in thermal equilibrium with the non-relativistic Fermi-Dirac or Bose-Einstein distributions:

$$
\left\langle n\left(p, T_{h}\right)\right\rangle=\frac{1}{\exp \left[p^{2} /\left(2 m_{h} k T_{h}\right)-\mu^{\prime}\right] \pm 1} .
$$

We define

$$
\mathbb{V} \equiv \sqrt{\frac{3 k T_{h}}{m_{h} c^{2}}}, \quad \mu^{\prime} \equiv \frac{\mu}{k T_{h}} .
$$

Note that $\mathbb{V}$ is proportional to $a^{-1}$, and is independent of the galaxy dark matter halo radial coordinate $r$. Note that $\mu^{\prime}$ is independent of $a$, but depends on $r$ (it becomes more negative with increasing $r$ ). We define

$$
\Sigma_{\mathrm{f}, \mathrm{b}} \equiv \frac{4}{\pi^{1 / 2}} \int_{0}^{\infty} \frac{y^{2} \mathrm{~d} y}{\exp \left[y^{2}-\mu^{\prime}\right] \pm 1}, \quad B_{\mathrm{f}, \mathrm{b}} \equiv \int_{0}^{\infty} \frac{y^{4} \mathrm{~d} y}{\exp \left[y^{2}-\mu^{\prime}\right] \pm 1} .
$$

Then, the density, mean-square velocity, and pressure are

$$
\rho_{h}=\frac{N_{\mathrm{f}, \mathrm{b}} m_{h}^{5 / 2}\left(k T_{h}\right)^{3 / 2} \Sigma_{\mathrm{f}, \mathrm{b}}}{2^{3 / 2} \pi^{3 / 2} \hbar^{3}}, \quad v_{h \mathrm{rms}}^{2}=\frac{8 k T_{h} B_{\mathrm{f}, \mathrm{b}}}{\pi^{1 / 2} m_{h} \Sigma_{\mathrm{f}, \mathrm{b}}}, \quad P_{h}=\frac{\rho_{h} v_{h \mathrm{rms}}^{2}}{3} .
$$

From these equations, applied to a homogeneous universe at the present time, we obtain

$$
m_{h}=\left[\frac{64 \pi^{3 / 4} \Omega_{c} \rho_{\text {crit }} \hbar^{3} B_{\mathrm{f}, \mathrm{b}}^{3 / 2}}{v_{h \mathrm{rms}}(1)^{3} N_{\mathrm{f}, \mathrm{b}} \Sigma_{\mathrm{f}, \mathrm{b}}^{5 / 2}}\right]^{1 / 4} .
$$

For fermions with $\mu^{\prime}=0$,

$$
\begin{gathered}
m_{h}=78.8\left(\frac{0.76 \mathrm{~km} / \mathrm{s}}{v_{h r \mathrm{~ms}}(1)}\right)^{3 / 4}\left(\frac{2}{N_{f}}\right)^{1 / 4} \mathrm{eV}, \\
\frac{T_{h}}{T}=0.392\left(\frac{v_{h \mathrm{rms}}(1)}{0.76 \mathrm{~km} / \mathrm{s}}\right)^{1 / 4}\left(\frac{2}{N_{f}}\right)^{1 / 4},
\end{gathered}
$$

where $T_{h} / T$ is the dark matter-to-photon temperature ratio after $\mathrm{e}^{+} \mathrm{e}^{-}$annihilation, and before dark matter becomes non-relativistic. For bosons with $\mu^{\prime}=-0.001$,

$$
\begin{gathered}
m_{h}=51.2\left(\frac{0.76 \mathrm{~km} / \mathrm{s}}{v_{h \mathrm{rms}}(1)}\right)^{3 / 4}\left(\frac{1}{N_{b}}\right)^{1 / 4} \mathrm{eV}, \\
\frac{T_{h}}{T}=0.511\left(\frac{v_{h \mathrm{rms}}(1)}{0.76 \mathrm{~km} / \mathrm{s}}\right)^{1 / 4}\left(\frac{1}{N_{b}}\right)^{1 / 4} .
\end{gathered}
$$

\section{Appendix B: Non-Relativistic Dark Matter Retaining the Ultra-Relativistic Momentum Distribution (URTE)}

In this Section we consider non-relativistic dark matter in thermal equilibrium 
with the ultra-relativistic Fermi-Dirac or Bose-Einstein distributions:

$$
\left\langle n\left(p, T_{h}\right)\right\rangle=\frac{1}{\exp \left[p c /\left(k T_{h}\right)-\mu^{\prime}\right] \pm 1} .
$$

Consider dark matter that is in thermal equilibrium with the standard model sector in the early universe, and decouples (from the standard model sector and from self interactions) while still ultra-relativistic. In this case, the number of dark matter particles per orbital remains unchanged during the transition to a non-relativistic gas. In this Appendix we assume that the dark matter-dark matter elastic interaction cross-section is sufficiently small that dark matter does not reach NRTE in the age of the universe. We define

$$
A_{\mathrm{f}, \mathrm{b}} \equiv \frac{1}{2 \pi^{2}} \int_{0}^{\infty} \frac{x^{2} \mathrm{~d} x}{\exp \left[x-\mu^{\prime}\right] \pm 1}, \quad C_{\mathrm{f}, \mathrm{b}} \equiv \int_{0}^{\infty} \frac{x^{4} \mathrm{~d} x}{\exp \left[x-\mu^{\prime}\right] \pm 1} .
$$

Then, the density, mean-square velocity, and pressure of the non-relativistic gas with the momentum distribution corresponding to ultra-relativistic thermal equilibrium (URTE), are

$$
\rho_{h}=m_{h} N_{\mathrm{f}, \mathrm{b}} A_{\mathrm{f}, \mathrm{b}}\left(\frac{k T_{h}}{\hbar c}\right)^{3}, \quad v_{h \mathrm{rms}}^{2}=\frac{C_{\mathrm{f}, \mathrm{b}}}{2 \pi^{2} A_{\mathrm{f}, \mathrm{b}}}\left(\frac{k T_{h}}{m_{h} c}\right)^{2}, \quad P_{h}=\frac{\rho v_{h \mathrm{rms}}^{2}}{3} .
$$

Note that $T_{h} \propto 1 / a, \rho_{h} \propto 1 / a^{3}, \quad v_{h r m s} \propto 1 / a$, and $P_{h} \propto 1 / a^{5}$. From these equations, applied to a homogeneous universe at the present time, we obtain

$$
m_{h}=\left[\frac{\Omega_{c} \rho_{\text {crit }} \hbar^{3} C_{\mathrm{f}, \mathrm{b}}^{3 / 2}}{2^{3 / 2} \pi^{3} v_{h r \mathrm{rms}}(1)^{3} N_{\mathrm{f}, \mathrm{b}} A_{\mathrm{f}, \mathrm{b}}^{5 / 2}}\right]^{1 / 4} .
$$

For fermions with $\mu^{\prime}=0$,

$$
\begin{gathered}
m_{h}=111\left(\frac{0.76 \mathrm{~km} / \mathrm{s}}{v_{h \mathrm{rms}}(1)}\right)^{3 / 4}\left(\frac{2}{N_{f}}\right)^{1 / 4} \mathrm{eV}, \\
\frac{T_{h}}{T}=0.333\left(\frac{v_{h \mathrm{rms}}(1)}{0.76 \mathrm{~km} / \mathrm{s}}\right)^{1 / 4}\left(\frac{2}{N_{f}}\right)^{1 / 4} .
\end{gathered}
$$

For bosons with $\mu^{\prime}=-0.001$,

$$
\begin{gathered}
m_{h}=113\left(\frac{0.76 \mathrm{~km} / \mathrm{s}}{v_{h \mathrm{rms}}(1)}\right)^{3 / 4}\left(\frac{1}{N_{b}}\right)^{1 / 4} \mathrm{eV}, \\
\frac{T_{h}}{T}=0.379\left(\frac{v_{h \mathrm{rms}}(1)}{0.76 \mathrm{~km} / \mathrm{s}}\right)^{1 / 4}\left(\frac{1}{N_{b}}\right)^{1 / 4} .
\end{gathered}
$$

Let us recall [4] that the pressure $P_{h}$ of the collisional or collision-less gas in Equation (5) is defined in (21) with

$$
\frac{v_{h r \mathrm{~ms}}^{2}}{3}=v_{r h \mathrm{rms}}^{2} \equiv\left\langle v_{r h}^{2}\right\rangle \equiv \frac{\int_{0}^{\infty} v_{r h}^{2} f\left(v_{r h}^{2}\right) \mathrm{d}\left(v_{r h}^{2}\right)}{\int_{0}^{\infty} f\left(v_{r h}^{2}\right) \mathrm{d}\left(v_{r h}^{2}\right)},
$$

where $f\left(v_{r h}^{2}\right) \mathrm{d}\left(v_{r h}^{2}\right)$ is proportional to the number of dark matter particles with $v_{r h}^{2}$ between $v_{r h}^{2}$ and $v_{r h}^{2}+\mathrm{d}\left(v_{r h}^{2}\right)$ with $v_{r h}>0$. 